\title{
Alterung des Gehirns: Charakterisierung mittels MRT
}

\author{
Wegener, S
}

\begin{abstract}
Unser Gehirn verändert sich mit zunehmendem Alter. Dieser physiologische Prozess kann mithilfe von Magnetresonanztomographie (MRT) beschrieben werden. Im Laufe des Lebens kommt es zu Atrophie (Schrumpfen von Hirnstrukturen) sowie Auftreten von charakteristischen MRT-SignalHyperintensitäten in der weissen Substanz. Eine besonders starke oder frühe Ausprägung dieser Veränderungen kann pathologisch sein. Die Abgrenzung zwischen gesundem Altern und Prozessen mit Krankheitswert ist dabei nicht einfach. In diesem Mini-Review sollen normale Alterungsprozesse des Gehirns beschrieben und krankhafte Veränderungen aufgezeigt werden, die weiterer Abklärung und Behandlung bedürfen.
\end{abstract}

DOI: https://doi.org/10.1024/1661-8157/a002661

Posted at the Zurich Open Repository and Archive, University of Zurich ZORA URL: https://doi.org/10.5167/uzh-135784

Journal Article

Accepted Version

Originally published at:

Wegener, S (2017). Alterung des Gehirns: Charakterisierung mittels MRT. Praxis, 106(9):477-81.

DOI: https://doi.org/10.1024/1661-8157/a002661 


\section{Praxis}

\section{Alterung des Gehirns: Charakterisierung mittels MRT --Manuskript-Entwurf--}

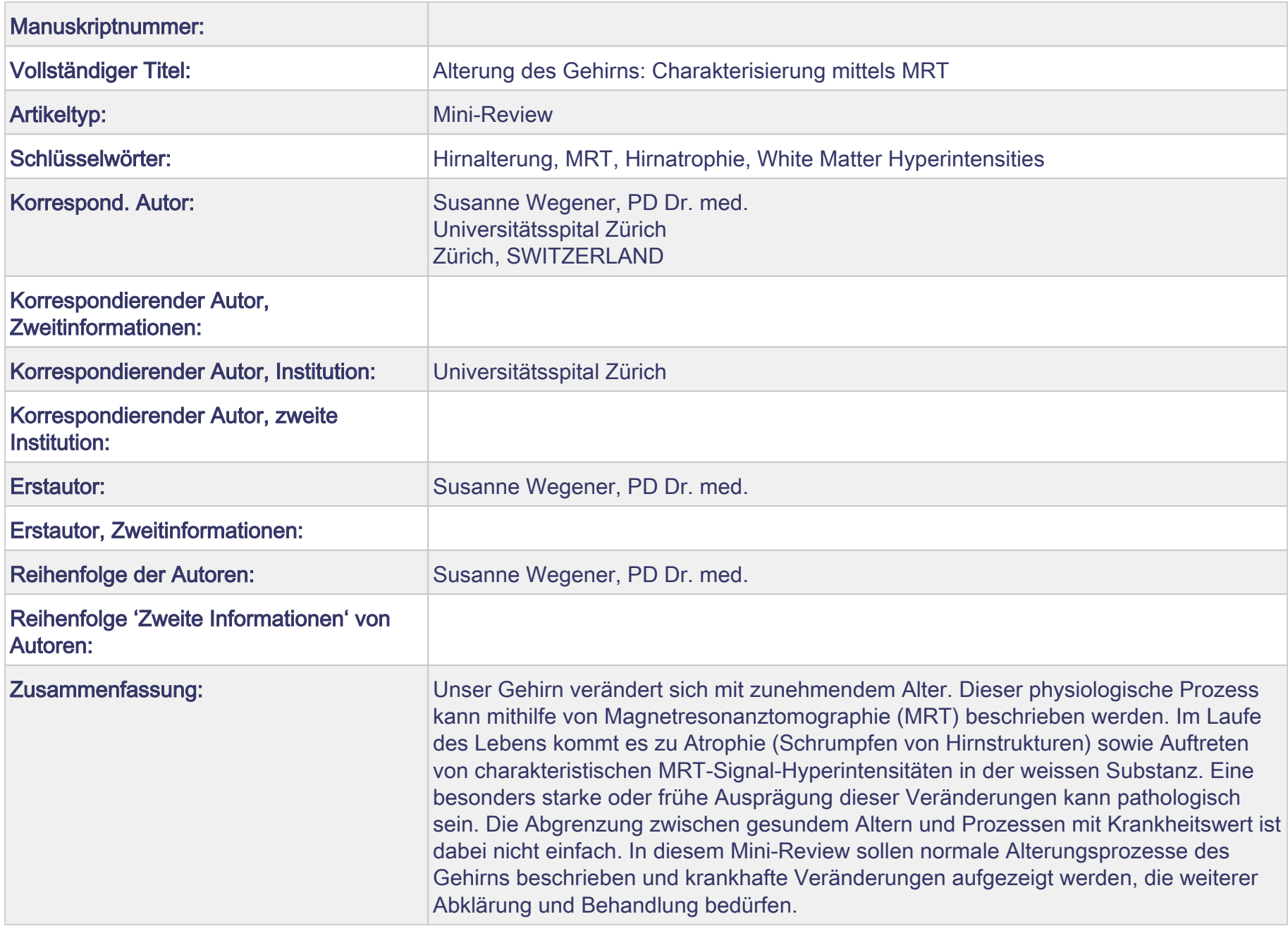




\section{Alterung des Gehirns: Charakterisierung mittels MRT Susanne Wegener \\ Klinik für Neurologie Universitätsspital Zürich}

«The Aging Brain: Imaging Correlates on MRI» 


\section{Abkürzungen}

EKG Elektrokardiogramm

MRT Magnetresonanztomographie

WMH White Matter Hyperintensities

$\mathrm{MCl}$ Mild Cognitive Impairment 


\title{
Zusammenfassung
}

Unser Gehirn verändert sich mit zunehmendem Alter. Dieser physiologische Prozess kann mithilfe von Magnetresonanztomographie (MRT) beschrieben werden. Im Laufe des Lebens kommt es zu Atrophie (Schrumpfen von Hirnstrukturen) sowie Auftreten von charakteristischen MRT-Signal-Hyperintensitäten in der weissen Substanz. Eine besonders starke oder frühe Ausprägung dieser Veränderungen kann pathologisch sein. Die Abgrenzung zwischen gesundem Altern und Prozessen mit Krankheitswert ist dabei nicht einfach. In diesem Mini-Review sollen normale Alterungsprozesse des Gehirns beschrieben und krankhafte Veränderungen aufgezeigt werden, die weiterer Abklärung und Behandlung bedürfen.

\begin{abstract}
Summary
Our brain changes as we get older. This process of physiological aging can be characterized by Magnetic Resonance Imaging (MRI). Shrinking of brain substance (atrophy) and development of lesions within the brain white matter can be observed. If such changes are particularly pronounced or occurring very early in life they may resemble pathological aging. The discrimination between normal aging and a disease process can be challenging. In this mini-review, our goal is to describe brain changes in healthy aging and point out pathological changes of the brain, which warrant further diagnostics and therapy.
\end{abstract}

\section{Zusammenfassung Französisch}

Schlüsselwörter:

Hirnalterung, MRT, Hirnatrophie, White Matter Hyperintensities

Brain aging, MRI, brain atrophy, white matter hyperintensities 


\section{1) Veränderungen des Gehirns im Alter}

\subsection{Einleitung}

Altern ist ein natürlicher, lebenslanger Prozess, der Veränderungen des Gehirns einschliesst. Hierzu gehören Entwicklung und Wachstum in der Jugend, aber auch Schrumpfen von Hirnstrukturen im Laufe des Erwachsenenlebens (1, 2). Gewisse daraus resultierende kognitive Leistungseinbussen sind Teil des normalen Alterns (4, 5). Neben physiologischen Veränderungen nimmt aber im Alter auch die Häufigkeit medizinischer Probleme und vaskulärer Risikofaktoren wie Bluthochdruck und Diabetes Typ II zu (6). Diese Begleiterkrankungen und andere Umwelteinflüsse, aber auch genetische Faktoren können dazu führen, dass Alterungsprozesse des Gehirns beschleunigt ablaufen. Die häufigsten strukturellen Veränderungen des Gehirns im Alter sind Atrophie, Veränderungen der weissen Substanz in Form von T2Hyperintensitäten (White Matter Hyperintensities: WMH) sowie stumme Hirninfarkte und Mikroblutungen.

\subsection{Atrophie}

Das Hirnvolumen nimmt beim Erwachsenen um 0.2-0.5\% pro Jahr ab (7). Der Abbau von Hirnsubstanz wird als Atrophie bezeichnet. Das Gehirn wird leichter; die Ventrikel und die Hirnfurchen (Sulci) erweitern sich (8). Diesen Veränderungen liegen der Untergang von Nervenzellen und myelinisierten Fasern, Veränderung von Anzahl und Aufbau synaptischer Strukturen sowie die Rückbildung von Blutgefässen zugrunde ( 9 , 10). Verschiedene Hirnregionen sind hiervon unterschiedlich stark betroffen: der präfrontale Kortex atrophiert bereits ab dem 20. Lebensjahr, während das Kleinhirn erst ab dem 50. Lebensjahr, dafür aber schneller an Volumen abnimmt $(11,12)$. Die insgesamt deutlichste Atrophie ist im Frontallappen zu verzeichnen (1). Atrophie des Gehirns lässt sich mit hochaufgelösten MRT-Techniken gut darstellen. Hierbei werden häufig dreidimensionale T1- gewichtete Aufnahmen verwendet. Mithilfe von DiffusionsTensor-Bildgebung (DTI) ist es sogar möglich, anatomische Verbindungen innerhalb des Gehirns darzustellen und zu quantifizieren (13). Auch die weisse Substanz zeigt eine Atrophie, beginnend um das 20. Lebensjahr, mit einer frontalen Betonung, die nicht unbedingt mit einer messbaren Reduktion des Hirnvolumens einhergehen muss (14). 
Beschleunigte Hirnatrophie kann unterschiedliche Gründe haben. Einerseits können Hirnstrukturen im Rahmen von neurodegenerativen Erkrankungen wie Demenzen sukzessive zugrunde gehen. So ist die Alzheimer-Demenz durch eine Atrophie des Temporallappens und des temporo-parietalen Cortex gekennzeichnet (15). Andererseits sind vaskuläre Risikofaktoren wie hoher Blutdruck und Adipositas ebenfalls mit einer beschleunigten (eher globalen) Hirnvolumenminderung assoziiert (16).

Für den praktischen Arzt stellt sich häufig die Frage, wie bei einem Patienten mit einer im MRT beschriebenen Hirnatrophie umzugehen ist, wenn dieser Befund nicht gezielt zur Abklärung einer Demenz erfolgte. Kann aufgrund einer im MRT dargestellten Atrophie die Diagnose einer Demenz gestellt werden? Der Begriff Demenz umschreibt einen Zustand, bei dem ein Mensch aufgrund von progredienten kognitiven Leistungseinbussen nicht mehr in der Lage ist, seinen gewohnten Alltagsfunktionen nachzukommen. Demenzen können pathophysiologisch verschiedene Ursachen haben und klinisch völlig unterschiedlich ausgeprägt sein. Etwa 20 Prozent der über 85 jährigen sind von einer Demenz betroffen (17). Häufige Ursachen sind neben dem Morbus Alzheimer die vaskuläre Demenz, Lewy-Körperchen- und Frontotemporale Demenz. Je nach Art der dementiellen Erkrankung, sind Ausmass und Lokalisation der Hirnatrophie unterschiedlich. Vor der Diagnose einer Demenz müssen andere Erkrankungen wie Delir oder Depression als Ursache der kognitiven Störung ausgeschlossen werden. Wichtig ist also: die Demenz ist eine Syndrom-Diagnose, die in erster Linie klinisch zu stellen ist. Wenn in einem MRT-Befund eine deutliche, über das Altersmass hinausgehende Atrophie festgestellt wurde, sollten Risikofaktoren eruiert und eine detaillierte Eigen- und Fremdanamnese bezüglich kognitiver Störungen erhoben werden. Eine Demenz sollte allerdings nicht einzig aufgrund eines MRT-Befundes diagnostiziert werden.

Eine besondere Rolle zwischen normalem Altern und Demenz nimmt die leichte kognitive Beeinträchtigung («Mild Cognitive Impairment, $M C l »)$ ein. Die Diagnosekriterien für $\mathrm{MCl}$ sind nicht ganz ein einheitlich: zusammengefasst haben Menschen mit $\mathrm{MCl}$ haben zwar gewisse kognitive Beeinträchtigungen, diese sind aber nicht schwer genug, um zu alltagsrelevanten Defiziten zu führen (18). Patienten mit $\mathrm{MCl}$ konvertieren nicht zwangsläufig zu einer Demenz; im Gegenteil: viele bleiben lange Zeit auf gutem Niveau kognitiv stabil (19). Auch für die Entität MCI wurden mit 
MRT Korrelate gesucht. Dies vor dem Hintergrund, Patienten mit einem hohen Demenzrisiko zu identifizieren und potentiellen Behandlungen zuführen zu können. So konnte hier eine Abnahme von Faserstrukturen im Bereich der Fornix identifiziert werden (20). Es gilt für $\mathrm{MCl}$ wie für die Demenz: entscheidend sind die klinischen Symptome und die Beschwerden des Patienten. Mögliche Empfehlungen zur Verminderung von Hirnvolumenabnahme durch normale oder pathologisch beschleunigte Alterungsprozesse sind ähnlich: ein möglichst aktiver körperlicher und geistiger Lebensstil können sich positiv auf die kognitive Leistungsfähigkeit und möglicherweise auch auf die im MRT nachweisbare Hirnatrophie auswirken (21).

\subsection{White Matter Hyperintensities (WMH)}

Neben Atrophie von Hirnstrukturen findet man im MRT des älteren Menschen häufig Veränderungen der subkortikalen, weissen Substanz, die als White Matter Hyperintensities (WMH) bezeichnet werden. Zur Darstellung werden in erster Linie FLAIR (fluid attenuated inversion recovery) Sequenzen verwendet, bei denen das Signal von Nervenwasser unterdrückt wird. So können auch kleinste Läsionen im Gehirn sichtbar gemacht werden können (Abbildung 1).

Wie viele und welche Art von $\mathrm{WMH}$ ist nun mit normalem Altern vereinbar und was geht darüber hinaus? Unterschiedliche Muster von WMH können nach Lokalisation und Form unterschieden werden: periventrikuläre und subkortikale (tiefe) WMH. Die verschiedenen Formen und Schweregrade der Veränderungen und deren pathophysiologische Mechanismen wurden von Schmidt et al. umfassend zusammengefasst (22). Periventrikuläre $\mathrm{WMH}$ sind besonders häufig an den Vorderhörnern der Seitenventrikel zu erkennen. In geringer Ausprägung bezeichnet man sie als «Kappen», in mittlerer Ausprägung als «Stift-dünne» Linien entlang der Seitenventrikel und wenn sehr deutlich und flächig vorhanden als «Halo». Subkortikale (tiefe) WMH sind initial punktuell, dann beginnend konfluierend und in voller Ausprägung konfluierend (22).

Einen Einblick in den natürlichen Verlauf von WMH bei älteren Menschen gab die Österreichische Schlaganfall-Präventions-Studie (3), Abbildung 2. 
WMH sind häufig bei gesunden Personen im Alter von 45-87 Jahren (Abbildung 2). In der Österreichischen Schlaganfall-Präventionsstudie konnten bei über der Hälfte der gesunden < 55-jährigen Menschen periventrikuläre Kappen und punktuelle tiefsubkortikale WMH nachgewiesen werden (3). Das Ausmass dieser Veränderungen stieg mit dem Alter. Halo-artige periventrikuläre oder konfluierende tief-subkortikale WMH sind bei Gesunden unter 65 sehr selten. Risikofaktoren wie arterielle Hypertonie, Diabetes mellitus oder Nikotinabusus sind gehäuft bei Menschen mit ausgeprägten WMH zu finden. Übrigens ist auch Atrophie, besonders frontal betonte, stärker betont, wenn vaskuläre Risikofaktoren vorliegen (23). Die Zunahme von WMH geht mit einem höheren Risiko für spätere kognitive Beeinträchtigung einher (24). Aber auch andere wichtige klinische Symptome wie Mobilität (durch eine Veränderung des Gangbildes), Inkontinenz und Depression wurden mit Ausmass und Progress von WMH assoziiert $(25,26)$. Weit fortgeschrittene WMH zeigten in einer anderen Erhebung bei ansonsten gesunden älteren Menschen einen schnelleren Übergang in funktionelle Abhängigkeit an (27).

Die histologische Charakterisierung von Veränderungen der weissen Substanz ergab, dass periventrikuläre WMH eher nicht-ischämischer Natur sind. Hierbei kommt es aufgrund einer fortschreitenden Blut-Hirn-Schrankenstörung in Ventrikelnähe zu einer Akkumulation von Liquor, dem Untergang von weisser Substanz und einer Einwanderung von Entzündungszellen (28, 29). Bei punktuellen tief-subkortikalen WMH handelt es sich meist um erweiterte perivaskuläre Räume, oder «Virchow-RobinRäume», mit interstitieller Flüssigkeit gefült, die mit dem subpialen Raum kommunizieren. Wahrscheinlich sind sie im Alter infolge von Funktionsstörungen kleinster, perforierender Hirngefässe erweitert (30). Als Ursache von konfluierenden, stärker ausgeprägten WMH muss hingegen von einer ischämischen Genese ausgegangen werden. Es zeigen sich in diesen Regionen Untergang von Nervenfasern und Gliose bis hin zu manifesten Infarkten (22).

Ist es möglich, in das Fortschreiten von WMH einzugreifen? Dies ist zumindest für eine konsequente Blutdruckeinstellung gezeigt worden (31). Auch körperliche Aktivität und sogar kognitives Training können möglicherweise den Progress von WMH bremsen (32). 
Somit sind WMH differenziert zu betrachten und je nach Ausprägung und Alter unterschiedlich $\mathrm{zu}$ interpretieren. Es ist sicher sinnvoll, bei Vorhandensein ausgeprägter WMH vaskuläre Risikofaktoren zu überprüfen und einzustellen.

\section{2) Pathologische Hirnalterungsprozesse: Stumme Hirninfarkte und Mikroblutungen}

Die bisher skizzierten Veränderungen des Gehirns sind als normal anzusehen, wenn sie nicht ein Altersübliches Ausmass überschritten haben. Stumme Hirninfarkte und Mikroblutungen sind dagegen Teil eines pathologischen Spektrums von Hirnalterungsprozessen. Mikroblutungen sind oft ähnlich lokalisiert wie tiefsubkortikale $\mathrm{WMH}$, und entstehen wahrscheinlich aufgrund vergleichbarer pathologischer Kleingefässprozesse und vaskulärer Risikofaktorkonstellationen. Sie werden mithilfe von $\mathrm{T}^{*}{ }^{*}$-gewichteten Sequenzen im MRT sichtbar gemacht. Bei Detektion von Mikroblutungen im Gehirn ist die Kontrolle des Blutdrucks entscheidend um weitere hypertensive Mikroblutungen oder gar klinisch manifeste Hirnparenchymblutungen zu verhindern. Lobäre Mikroblutungen wiederum können eine Amyloidangiopathie anzeigen (33). Menschen mit vielen Mikroblutungen, besonders in lobärer Lage, haben ein deutlich erhöhtes Risiko für Hirnblutungen durch Medikamente wie Antikoagulantien (34).

Ischämische Hirninfarkte können so ablaufen, dass der Betroffene keine Symptome bemerkt. Sie werden dann als «stumme» Hirninfarkte bezeichnet. Wenngleich in der Akutphase klinisch stumm, beschleunigen sie pathologische Alterungsprozesse des Gehirns und führen zu ähnlichen Symptomen wie ausgeprägte $\mathrm{WMH}$ : Depression, Verlust kognitiver Fähigkeiten, Gangstörungen. Im Gegensatz zu WMH sind diese Symptome eher "Stufenartig» verstärkt, nicht schleichend progredient. Stumme Hirninfarkte lassen sich als Zufallsbefunde bei ca. 4-8\% der 50-60-jährigen und 25$40 \%$ der über 80 -jährigen nachweisen (35). Stumme Hirninfarkte sind oft lakunär (mikroangiopathisch verursacht, subkortikal gelegen) und mit WMH assoziiert (36). Einen Sonderfall stellt die genetische Disposition zur Kleingefäss-Ischämie dar, wie bei der CADASIL-Erkrankung (CADASIL = Cerebral Autosomal Dominant Arteriopaty with Subcortical Infarcts and Leucencephalopathy), wo es aufgrund einer Mutation im NOTCH-3-Gen zu wiederholten Ischämien und progredienten WMH kommt (Abbildung 
3). Die Diagnose erfolgt bei ausreichendem Verdacht aufgrund klinischer Symptome und Bildgebung mithilfe einer gezielten genetischen Untersuchung (37).

\section{3)Therapeutische Konsequenzen von MRT-Veränderungen des Gehirns im Alter}

Aufgrund einer nur moderaten Risikoreduktion kardiovaskulärer Ereignisse bei signifikant erhöhtem Blutungsrisiko kann die routinemässige Anwendung von Aspirin zur Primärprophylaxe bei älteren Menschen zur Zeit nicht empfohlen werden (38, 39). Wenn im MRT ein klinisch stummer Hirninfarkt oder über das Altersmass hinausgehende $\mathrm{WMH}$ nachgewiesen werden, sollten gründliche Abklärungen zur möglichen Ätiologie durchgeführt werden. Diese schliessen eine Duplexuntersuchung der hirnzuführenden Gefässe, die Suche nach kardialer Embolie-Quelle mit LangzeitEKG und Echokardiographie sowie die Suche nach vaskulären Risikofaktoren (Diabetes, Dyslipidämie, 24h-Blutdruck-Messung) ein. Im Falle eines stummen Hirninfarktes sollte je nach Befund der Abklärungen eine geeignete Sekundärprophylaxe für den Patienten ausgewählt werden. Bei WMH stehen die Kontrolle der vaskulären Risikofaktoren und die Motivation zu einem körperlich und geistig aktiven Lebensstil im Vordergrund. 


\section{4) Referenzen}

1. DeCarli C, Massaro J, Harvey D, Hald J, Tullberg M, Au R, et al. Measures of brain morphology and infarction in the framingham heart study: establishing what is normal. Neurobiol Aging. 2005;26(4):491-510.

2. Rushton JP, Ankney CD. Brain size and cognitive ability: Correlations with age, sex, social class, and race. Psychon Bull Rev. 1996;3(1):21-36.

3. Schmidt R, Lechner H, Fazekas F, Niederkorn K, Reinhart B, Grieshofer P, et al. Assessment of cerebrovascular risk profiles in healthy persons: definition of research goals and the Austrian Stroke Prevention Study (ASPS). Neuroepidemiology. 1994;13(6):308-13.

4. Josefsson M, de Luna X, Pudas S, Nilsson LG, Nyberg L. Genetic and lifestyle predictors of 15-year longitudinal change in episodic memory. J Am Geriatr Soc. 2012;60(12):2308-12.

5. Salthouse TA. The processing-speed theory of adult age differences in cognition. Psychol Rev. 1996;103(3):403-28.

6. Vasan RS, Levy D. Rates of progression to hypertension among nonhypertensive subjects: implications for blood pressure screening. Eur Heart $\mathrm{J}$. 2002;23(14):1067-70.

7. Fjell AM, McEvoy L, Holland D, Dale AM, Walhovd KB, Alzheimer's Disease Neuroimaging I. What is normal in normal aging? Effects of aging, amyloid and Alzheimer's disease on the cerebral cortex and the hippocampus. Prog Neurobiol. 2014;117:20-40.

8. Raz N, Rodrigue KM. Differential aging of the brain: patterns, cognitive correlates and modifiers. Neurosci Biobehav Rev. 2006;30(6):730-48.

9. Pakkenberg B, Gundersen HJ, Mortensen EL, Lauritzen MJ, Jeune B, Regeur L, et al. [The normal brain: a new knowledge in different fields]. Ugeskr Laeger. 1997;159(6):723-7.

10. Liu X, Erikson C, Brun A. Cortical synaptic changes and gliosis in normal aging, Alzheimer's disease and frontal lobe degeneration. Dementia. 1996;7(3):128-34.

11. Raz N, Gunning FM, Head D, Dupuis JH, McQuain J, Briggs SD, et al. Selective aging of the human cerebral cortex observed in vivo: differential vulnerability of the prefrontal gray matter. Cereb Cortex. 1997;7(3):268-82 . 
12. Luft AR, Skalej M, Schulz JB, Welte D, Kolb R, Burk K, et al. Patterns of agerelated shrinkage in cerebellum and brainstem observed in vivo using threedimensional MRI volumetry. Cereb Cortex. 1999;9(7):712-21.

13. Moseley M. Diffusion tensor imaging and aging - a review. NMR Biomed. 2002;15(7-8):553-60.

14. Sullivan EV, Pfefferbaum A. Diffusion tensor imaging and aging. Neurosci Biobehav Rev. 2006;30(6):749-61.

15. Seeley WW, Crawford RK, Zhou J, Miller BL, Greicius MD. Neurodegenerative diseases target large-scale human brain networks. Neuron. 2009;62(1):42-52.

16. Nettiksimmons J, Beckett L, Schwarz C, Carmichael O, Fletcher E, Decarli C. Subgroup of ADNI normal controls characterized by atrophy and cognitive decline associated with vascular damage. Psychol Aging. 2013;28(1):191-201.

17. Prince M, Bryce R, Albanese E, Wimo A, Ribeiro W, Ferri CP. The global prevalence of dementia: a systematic review and metaanalysis. Alzheimers Dement. 2013;9(1):63-75 e2.

18. Petersen RC. Mild cognitive impairment as a diagnostic entity. J Intern Med. 2004;256(3):183-94.

19. Petersen RC, Smith GE, Waring SC, Ivnik RJ, Tangalos EG, Kokmen E. Mild cognitive impairment: clinical characterization and outcome. Archives of neurology. 1999;56(3):303-8.

20. Fletcher E, Raman M, Huebner P, Liu A, Mungas D, Carmichael O, et al. Loss of fornix white matter volume as a predictor of cognitive impairment in cognitively normal elderly individuals. JAMA Neurol. 2013;70(11):1389-95.

21. Smith JC, Lancaster MA, Nielson KA, Woodard JL, Seidenberg M, Durgerian S, et al. Interactive effects of physical activity and APOE-epsilon4 on white matter tract diffusivity in healthy elders. Neuroimage. 2016;131:102-12.

22. Schmidt R, Schmidt H, Haybaeck J, Loitfelder M, Weis S, Cavalieri M, et al. Heterogeneity in age-related white matter changes. Acta Neuropathol. 2011;122(2):171-85.

23. Lockhart SN, DeCarli C. Structural imaging measures of brain aging. Neuropsychol Rev. 2014;24(3):271-89.

24. Carmichael O, Schwarz C, Drucker D, Fletcher E, Harvey D, Beckett L, et al. Longitudinal changes in white matter disease and cognition in the first year of the 
Alzheimer disease neuroimaging initiative. Archives of neurology. 2010;67(11):13708.

25. Qiu WQ, Himali JJ, Wolf PA, DeCarli DC, Beiser A, Au R. Effects of white matter integrity and brain volumes on late life depression in the Framingham Heart Study. Int J Geriatr Psychiatry. 2016.

26. Abraham HM, Wolfson L, Moscufo N, Guttmann CR, Kaplan RF, White WB. Cardiovascular risk factors and small vessel disease of the brain: Blood pressure, white matter lesions, and functional decline in older persons. J Cereb Blood Flow Metab. 2016;36(1):132-42.

27. Inzitari D, Pracucci G, Poggesi A, Carlucci G, Barkhof $F$, Chabriat $H$, et al. Changes in white matter as determinant of global functional decline in older independent outpatients: three year follow-up of LADIS (leukoaraiosis and disability) study cohort. BMJ. 2009;339:b2477.

28. Simpson JE, Ince PG, Higham CE, Gelsthorpe CH, Fernando MS, Matthews F, et al. Microglial activation in white matter lesions and nonlesional white matter of ageing brains. Neuropathol Appl Neurobiol. 2007;33(6):670-83.

29. Haller S, Kovari E, Herrmann FR, Cuvinciuc V, Tomm AM, Zulian GB, et al. Do brain T2/FLAIR white matter hyperintensities correspond to myelin loss in normal aging? A radiologic-neuropathologic correlation study. Acta neuropathologica communications. 2013;1:14.

30. Gess B, Niederstadt TU, Ringelstein EB, Schabitz WR. [Clinical relevance of normal and enlarged Virchow-Robin spaces]. Nervenarzt. 2010;81(6):727-33.

31. White WB, Petry NM. Home blood pressure monitoring as an intervention to control hypertension: comment on "Home blood pressure management and improved blood pressure control". Arch Intern Med. 2011;171(13):1181-2.

32. Wirth M, Haase CM, Villeneuve S, Vogel J, Jagust WJ. Neuroprotective pathways: lifestyle activity, brain pathology, and cognition in cognitively normal older adults. Neurobiol Aging. 2014;35(8):1873-82.

33. Greenberg SM, Vernooij MW, Cordonnier C, Viswanathan A, Al-Shahi Salman $\mathrm{R}$, Warach $\mathrm{S}$, et al. Cerebral microbleeds: a guide to detection and interpretation. Lancet Neurol. 2009;8(2):165-74.

34. Greenberg SM, Eng JA, Ning M, Smith EE, Rosand J. Hemorrhage burden predicts recurrent intracerebral hemorrhage after lobar hemorrhage. Stroke. 2004;35(6):1415-20. 
35. Vermeer SE, Longstreth WT, Jr., Koudstaal PJ. Silent brain infarcts: a systematic review. Lancet Neurol. 2007;6(7):611-9.

36. Ritter MA, Dittrich R, Ringelstein EB. [Silent brain infarcts]. Nervenarzt. 2011;82(8):1043-52.

37. Chabriat H, Joutel A, Dichgans M, Tournier-Lasserve E, Bousser MG. Cadasil. Lancet Neurol. 2009;8(7):643-53.

38. Guirguis-Blake JM, Evans CV, Senger CA, Rowland MG, O'Connor EA, Whitlock EP. Aspirin for the Primary Prevention of Cardiovascular Events: A Systematic Evidence Review for the US Preventive Services Task Force. U.S. Preventive Services Task Force Evidence Syntheses, formerly Systematic Evidence Reviews. Rockville (MD)2015.

39. Halvorsen S, Andreotti F, ten Berg JM, Cattaneo M, Coccheri S, Marchioli R, et al. Aspirin therapy in primary cardiovascular disease prevention: a position paper of the European Society of Cardiology working group on thrombosis. J Am Coll Cardiol. 2014;64(3):319-27. 


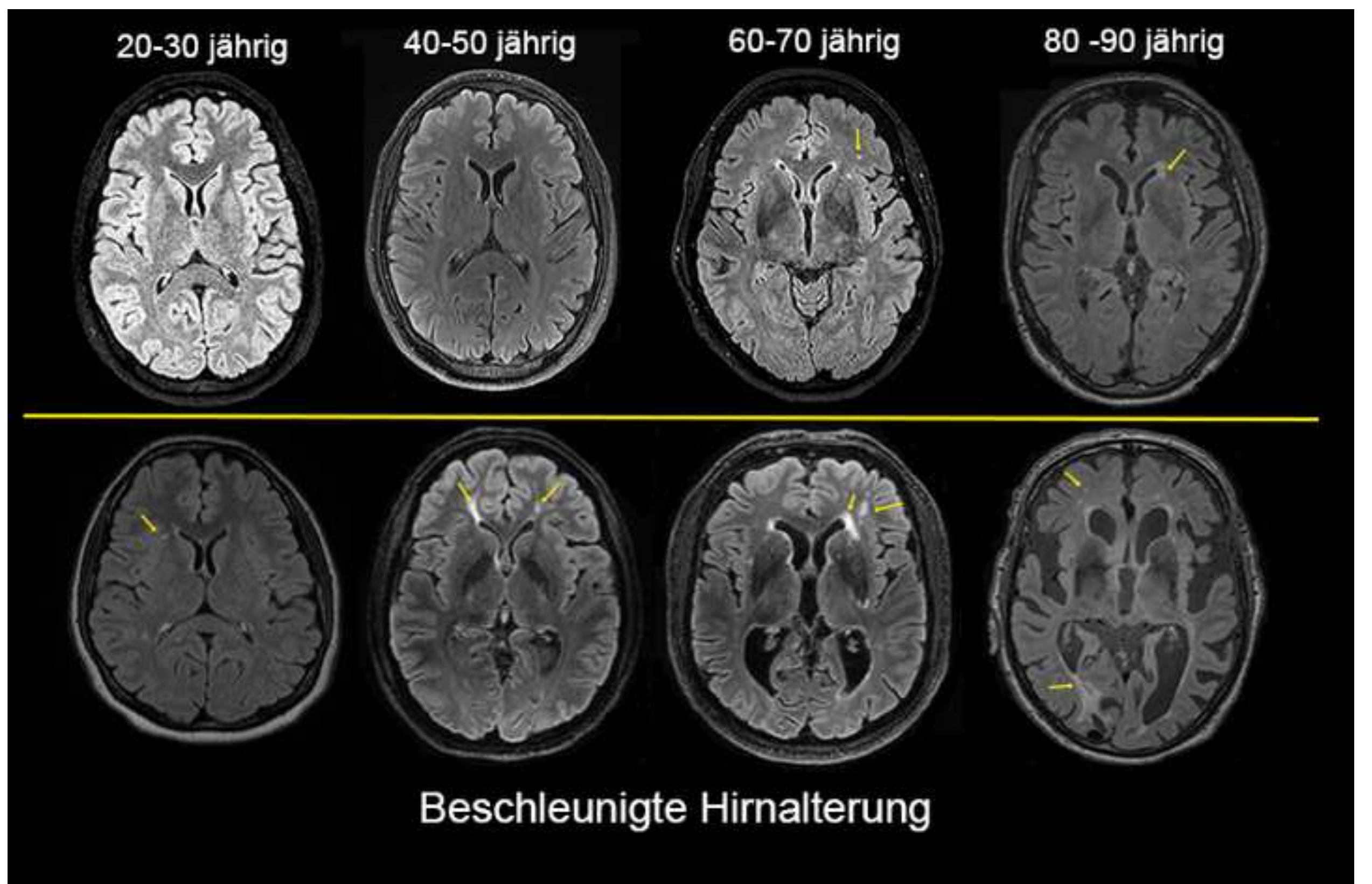




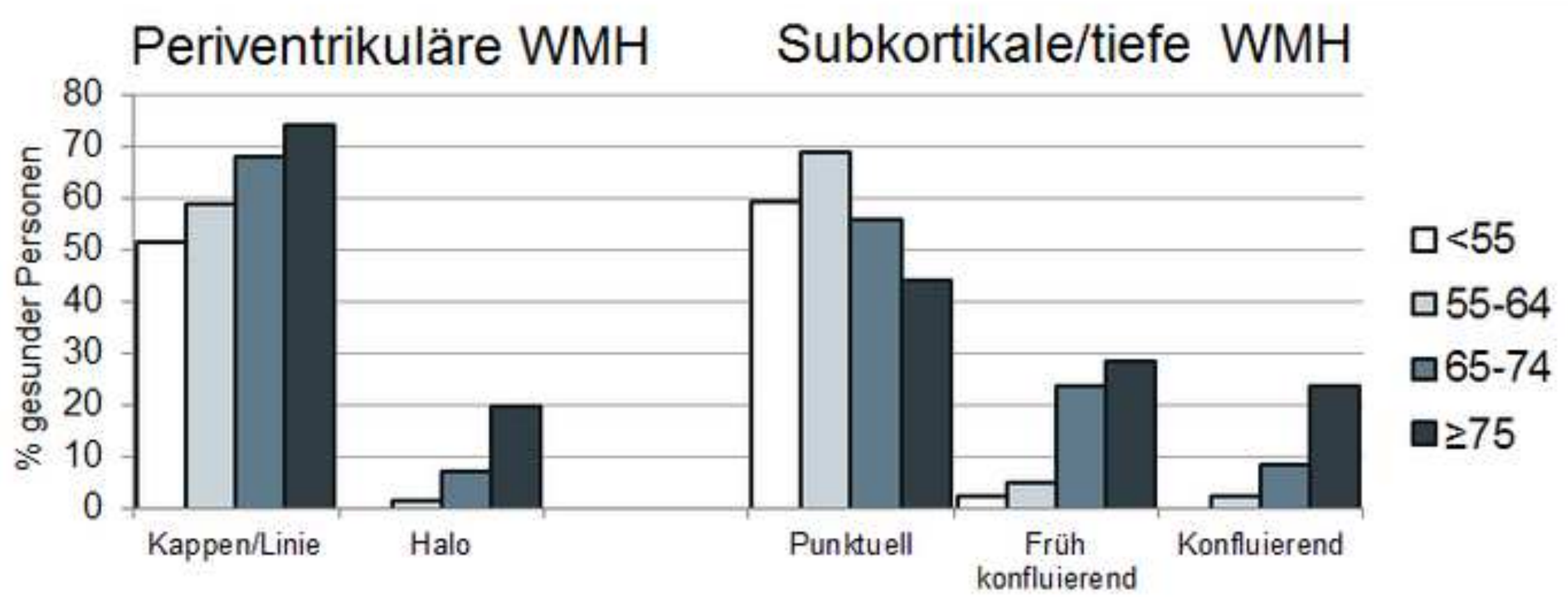



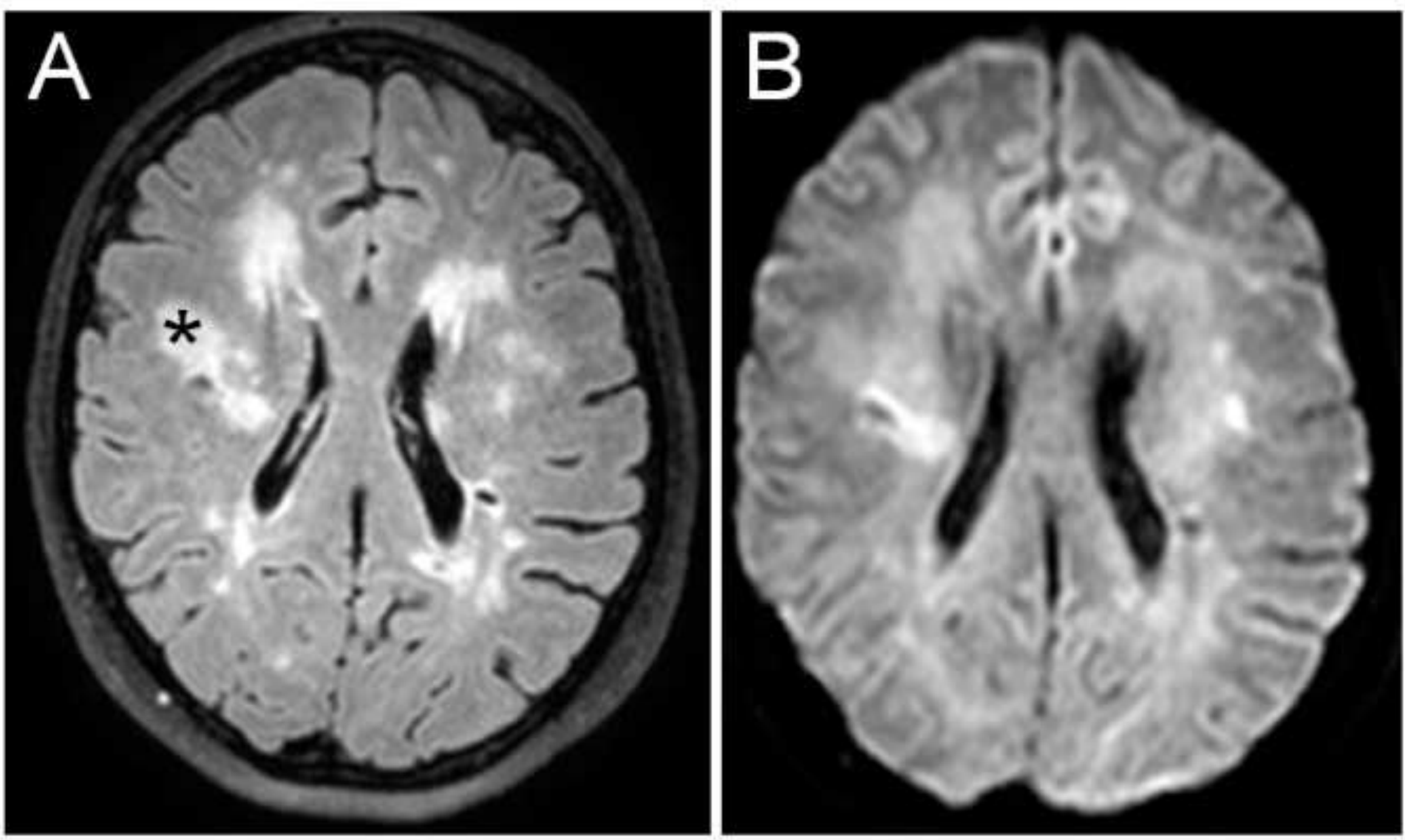


\section{5) Abbildungslegenden}

Abbildung 1: Veränderungen des Gehirns im Alter

FLAIR-MRTs gesunder Menschen unterschiedlichen Alters mit Altersentsprechenden (oberer Zeile) und über das Altersmass hinausgehenden (untere Zeile)

Veränderungen des Gehirns (Atrophie und WMH, letztere sind durch Pfeile hervorgehoben).

Mit Genehmigung der Klinik für Neuroradiologie, USZ.

Abbildung 2: Prävalenz unterschiedlicher WML in 993 Teilnehmern (45-87 Jahre) der Österreicher Schlaganfall Präventionsstudie*

Häufigkeit (in \%) unterschiedlicher WMH in den MRTs gesunder Teilnehmer unterschiedlichen Alters aus der Österreicher Schlaganfall Präventionsstudie (3). Bei mehreren Individuen waren unterschiedliche Arten von WMH vorhanden.

Abbildung 3: WMH und stumme Hirninfarkte in einer Patientin mit CADASIL

50-jährige Patientin mit gesicherter CADASIL-Erkrankung. A) FLAIR-MRT mit konfluierenden subkortikalen (tiefen) $\mathrm{WMH}\left({ }^{*}\right)$ und periventrikulären HALOCharakteristika. B) Diffusions-gewichtetes MRT (DWI) demonstriert einen frischen ischämischen Infarkt in subkortikaler (lakunärer) Konfiguration (Pfeil).

Mit Genehmigung der Klinik für Neuroradiologie, USZ 


\section{4) Key Messages}

1) Normale Veränderungen des Gehirns mit dem Alter sind Atrophie und das Auftreten von Hyperintensitäten der Weissen Substanz.

2) Stumme Hirninfarkte und Mikroblutungen gehören zu den pathologischen Hirnalterungserscheinungen.

3) Wenn Hirnalterung im MRT über das Altersmass hinausgeht, sollten Abklärungen bezüglich des Vorliegens einer kognitiven Störung und vaskulärer Risikofaktoren erfolgen. 


\section{Lernfragen:}

Sind subkortikale FLAIR-Hyperintensitäten in der weissen Substanz bei Menschen unter 60 Jahren pathologisch?
a) Immer.
b) $\mathrm{Ja}$, insbesondere, wenn sie ventrikelnah (periventrikulär) sind.
c) Punktuelle, tief-subkortikale und/oder gering ausgeprägte (Kappen-artige) periventrikuläre FLAIR-Hyperintensitäten können auch bei Gesunden unter 60 Jahren auftreten.
(c ist richtig)

Bei Nachweis einer deutlichen Hirnatrophie im MRT als Zufallsbefund:
a) Liegt eine Demenz vor.
b) Sollte man gezielt nach Symptomen einer dementiellen Entwicklung fragen.
c) Kann der Patient an einen Neurologen zur weiteren Abklärung und Beratung zugewiesen werden. (b und $c$ sind richtig) 


\section{Summary}

Our brain changes as we get older. This process of physiological aging can be characterized by Magnetic Resonance Imaging (MRI). Shrinking of brain substance (atrophy) and development of lesions within the brain white matter can be observed. If such changes are particularly pronounced or occurring very early in life they may resemble pathological aging. The discrimination between normal aging and a disease process can be challenging. In this mini-review, our goal is to describe brain changes in healthy aging and point out pathological changes of the brain, which warrant further diagnostics and therapy.

\section{Key points}

Brain aging, MRI, brain atrophy, white matter hyperintensities 


\section{Zusammenfassung}

Unser Gehirn verändert sich mit zunehmendem Alter. Dieser physiologische Prozess kann mithilfe von Magnetresonanztomographie (MRT) beschrieben werden. Im Laufe des Lebens kommt es zu Atrophie (Schrumpfen von Hirnstrukturen) sowie Auftreten von charakteristischen MRT-Signal-Hyperintensitäten in der weissen Substanz. Eine besonders starke oder frühe Ausprägung dieser Veränderungen kann pathologisch sein. Die Abgrenzung zwischen gesundem Altern und Prozessen mit Krankheitswert ist dabei nicht einfach. In diesem Mini-Review sollen normale Alterungsprozesse des Gehirns beschrieben und krankhafte Veränderungen aufgezeigt werden, die weiterer Abklärung und Behandlung bedürfen.

\section{Schlüsselwörter:}

Hirnalterung, MRT, Hirnatrophie, White Matter Hyperintensities 